OPEN ACCESS

Edited by:

Daya Shankar Gupta, Camden County College,

United States

Reviewed by:

Keita Mitani,

Tokyo Institute of Technology, Japan Hakan Karşilar,

Koç University, Turkey

*Correspondence:

Lihan Chen

CLH@pku.edu.cn

Received: 20 February 2018 Accepted: 16 May 2018

Published: 05 June 2018

Citation:

Wan Y and Chen L (2018) Temporal Reference, Attentional Modulation, and Crossmodal Assimilation. Front. Comput. Neurosci. 12:39. doi: 10.3389/fncom.2018.00039

\section{Temporal Reference, Attentional Modulation, and Crossmodal Assimilation}

\author{
Yingqi Wan and Lihan Chen* \\ School of Psychological and Cognitive Sciences and Beijing Key Laboratory of Behavior and Mental Health, Peking \\ University, Beijing, China
}

Crossmodal assimilation effect refers to the prominent phenomenon by which ensemble mean extracted from a sequence of task-irrelevant distractor events, such as auditory intervals, assimilates/biases the perception (such as visual interval) of the subsequent task-relevant target events in another sensory modality. In current experiments, using visual Ternus display, we examined the roles of temporal reference, materialized as the time information accumulated before the onset of target event, as well as the attentional modulation in crossmodal temporal interaction. Specifically, we examined how the global time interval, the mean auditory inter-intervals and the last interval in the auditory sequence assimilate and bias the subsequent percept of visual Ternus motion (element motion vs. group motion). We demonstrated that both the ensemble (geometric) mean and the last interval in the auditory sequence contribute to bias the percept of visual motion. Longer mean (or last) interval elicited more reports of group motion, whereas the shorter mean (or last) auditory intervals gave rise to more dominant percept of element motion. Importantly, observers have shown dynamic adaptation to the temporal reference of crossmodal assimilation: when the target visual Ternus stimuli were separated by a long gap interval after the preceding sound sequence, the assimilation effect by ensemble mean was reduced. Our findings suggested that crossmodal assimilation relies on a suitable temporal reference on adaptation level, and revealed a general temporal perceptual grouping principle underlying complex audio-visual interactions in everyday dynamic situations.

Keywords: temporal window, temporal ventriloquism effect, central tendency effect, assimilation, attention

\section{INTRODUCTION}

Multisensory interaction has been traditionally revealed to take place over a narrowed window time-i.e., within a presumed "temporal window" (Meredith et al., 1987; Powers et al., 2009; Vroomen and Keetels, 2010; Wallace and Stevenson, 2014; Gupta and Chen, 2016). For example, paired sound/tactile events presented in temporal proximity to paired visual events can alter the perceived interval between the visual stimuli, and hence bias the perception of visual apparent motion (Keetels and Vroomen, 2008; Chen et al., 2010; Shi et al., 2010). The above illusions have been typically known as temporal ventriloquism (Chen and Vroomen, 2013). Studies on temporal ventriloquism indeed suggested that crossmodal events appearing in temporal proximities have higher probabilities of "correlation" and even "causation" relations (Ernst and Di Luca, 2011; Parise et al., 2012). Based on those relations, sensory events with higher functional priorities (such 
as "precision" in timing) would calibrate/attract the counterpart events (with lower functional appropriateness) from the other modalities, give rise to successful multisensory integration. During the integration, multisensory events within a presumed short time window will largely obey the "assumption of unity," in which the coherent representation of multiple events become possible when they have been deemed as coming from a common source (Vatakis and Spence, 2007, 2008; Misceo and Taylor, 2011; Chuen and Schutz, 2016; Chen and Spence, 2017). As a result, the effectiveness of crossmodal interaction is enhanced.

However, the presumed "temporal window" for integration has often been violated in many ecological scenarios. Take an example: upon hearing the whistle of a running car behind us, after a decent long delay, we can know exactly what kind of the "car" is approaching and then make prompt avoidance. This indicates that humans can adaptively use the prior knowledge and employ the temporal/spatial information (including environmental cues associated with the sound) to facilitate the perceptual decision. This daily scenario, however, imposes a great challenge for human perception. How are perceptual grouping and correspondences between events achieved when the crossmodal events are separated both in longer temporal ranges and with larger temporal disparities? Moreover, for the longer temporal range, observers have difficulties in memorizing all the events and the processing of the sensory properties (including time information) would probably exceed their working memory capacities (Cowan, 2001; Klemen et al., 2009; Klemen and Chambers, 2012; Cohen et al., 2016). Therefore, the efficiency of crossmodal interaction will be reduced accordingly. The complex timing scenario as well as the challenge for time cognition also stems from the variance of the multiple time intervals. In short temporal range (such as around $2 \mathrm{~s}$ ), human observers could discriminate the short temporal intervals when the coefficient of variance (i.e., "CV," the ratio of the interval deviation to its baseline value) is less than 0.3 . The discrimination ability is greatly reduced when the $\mathrm{CV}$ is above 0.3 (Allan, 1974; Getty, 1975; Penney et al., 2000).

To cope with the above constraints, human observers adopt one of the efficient perceptual strategies-"ensemble coding" to process the mean properties of multiple events. For example, people can extract the mean rhythm of a given sound sequence and use this information to allocate visual attention and facilitate the detection of target events (Miller et al., 2013). Recent studies have shown that this averaging process is highly dependent on the temporal reference. The temporal reference included the generally global time interval before the onset of target event(s), the variabilities of the multiple intervals and the critical information of the last interval (Jones and McAuley, 2005; Acerbi et al., 2012; Cardinal, 2015; Karaminis et al., 2016). One compelling example is the central tendency effect within the broader framework of Bayesian optimization (Jazayeri and Shadlen, 2010; Shi et al., 2013; Shi and Burr, 2016; Roach et al., 2017), whereby incorporating the mean of the statistical distribution in the estimation would assimilate the estimates toward the mean (Jazayeri and Shadlen, 2010; Burr et al., 2013; Karaminis et al., 2016). For example, the estimation of a target property, such as the duration of an event, is assimilated toward to the mean duration of previously encountered target events (i.e., event history) (Nakajima et al., 1992; Burr et al., 2013; Shi et al., 2013; Roach et al., 2017). The central tendency effect indicates that human observers exploit predictive coding using the averaged sensory properties (Shi and Burr, 2016). The predictive coding framework states that the brain produces a Bayesian estimate of the environment (Friston, 2010). A strong mismatch between the prediction and the actual sensory input leads to an update of the internal model, and could trigger observable changes in perceptual decision. During this updating, attentional process can be considered as a form of predictive coding to establish an expectation of the moments in time until the task-relevant, to be integrated stimulus inputs arrive (Klemen and Chambers, 2012). On the other hand, the temporal reference (including temporal window) for crossmodal interaction is flexible by perceptual training (Powers et al., 2009, 2012), repeated exposure (adaptation) to the sensory stimuli (Mégevand et al., 2013), or recalibration process through experience (Sugano et al., 2010, 2012, 2016; Bruns and Röder, 2015; Habets et al., 2017). The flexibility of temporal window has also been shown to be shaped by the individual differences (Hillock et al., 2011; Stevenson et al., 2012, 2014; Lewkowicz and Flom, 2014; Chen et al., 2016; Hillock-Dunn et al., 2016).

Time perception is intrinsically related with attention and memory (Block and Gruber, 2014). Attention has been revealed to act as an essential cognitive faculty in integrating information in the multisensory mind (Duncan et al., 1997; Talsma et al., 2007, 2010; Donohue et al., 2011, 2015; Tang et al., 2016). (Selective) attention improves the efficiency of pooling task-relevant information - multiple (complex) properties (Buchan and Munhall, 2011; Li et al., 2016). Withdrawing attention has been shown in other tasks/paradigms to degrade the representation of individual sensory properties (Alsius et al., 2005, 2014). In the central tendency effect, observers processed task-relevant sensory properties to obtain the subsequent perceptual decision. However, whether/how attentional modulation would deplete the limited attentional resources for ensemble coding and hence play a role in the crossmodal assimilation, has not been empirically examined.

Therefore, in the present study, we aimed to examine how the temporal reference and the attentional processing would affect the crossmodal assimilation. We adopted "temporal ventriloquism effect" with visual Ternus display. We investigated how the temporal configurations between an auditory sequence (with multiple inter-intervals) and the visual Ternus display (with one interval) modulate the visual apparent-motion percepts. Ternus display can elicit two distinct percepts of visual apparent motion: "element" motion or "group" motion, determined by the visual inter-stimulus-interval (ISIV) between the two display frames (with other stimulus settings being fixed). Element motion is typically observed with short $\mathrm{ISI}_{\mathrm{V}}$ (e.g., of $50 \mathrm{~ms}$ ), and group motion with long ISIV (e.g., of $230 \mathrm{~ms}$ ) (Ternus, 1926; Shi et al., 2010) (see Supplement 1 for visual animation of Ternus display). Previously we have shown that when two beeps were presented in temporal proximity to, or synchronously with, the two visual frames respectively, the beeps can systematically bias the transitional threshold of visual apparent motion (Shi et al., 
2010). Here we extended the Ternus temporal ventriloquism paradigm to investigate the temporal crossmodal ensemble coding. We implemented five experiments to address this issue. Experiments 1 and 2 examined the role of temporal windowinterval gap between the offset of sound sequence and the onset of target Ternus display, to show the temporal constraints of central tendency effect. Experiment 3 compared the central tendency effect with the recency effect, by manipulating both the mean auditory interval and the last auditory interval. In Experiment 4, we fixed the last interval to be equal to the transitional threshold of perceiving element vs. group motion in the pretest, and manipulated the mean auditory inter-interval to show a genuine central tendency effect during crossmodal assimilation. In Experiment 5, we implemented dual-tasks and asked observers to perform the visual Ternus task while fulfilling a concurrent task of counting oddball sounds. Overall, the current results revealed that crossmodal central tendency effect is subject to the temporal reference (including the length of global time interval, the mean interval and the last interval for a given sound sequence) but less dependent on attentional modulation.

\section{MATERIALS AND METHODS}

\section{Participants}

A total of 60 participants $(14,13,7,12,14$ in Experiments $1-5)$, ages ranging from 18 to 33 years, took part in the main experiments. A post-hoc power estimation has shown the statistical powers are generally approaching or above 0.8 for the given sample sizes. All observers had normal or corrected-tonormal vision and reported normal hearing. The experiments were performed in compliance with the institutional guidelines set by the Academic Affairs Committee, School of Psychological and Cognitive Sciences, Peking University. The protocol was approved by the Committee for Protecting Human and Animal Subjects, School of Psychological and Cognitive Sciences, Peking University. All participants gave written informed consent in accordance with the Declaration of Helsinki, and were paid for their time on a basis of $40 \mathrm{CNY} /$ hour, i.e., $6.3 \mathrm{US}$ dollars/hour.

\section{Apparatus and Stimuli}

The experiments were conducted in a dimly lit (luminance: 0.09 $\mathrm{cd} / \mathrm{m}^{2}$ ) room. Visual stimuli were presented at the center of a $22-$ inch CRT monitor (FD 225P) at a screen resolution of $1024 \times 768$ pixels and a refresh rate of $100 \mathrm{~Hz}$. Viewing distance was $57 \mathrm{~cm}$, maintained by using a chin rest. A Ternus display consisted of two stimulus frames, each containing two black discs (10.30 $\mathrm{cd} / \mathrm{m}^{2}$; disc diameter and separation between discs: $1.6^{\circ}$ and $3^{\circ}$ of visual angle, respectively) presented on a gray background $\left(16.3 \mathrm{~cd} / \mathrm{m}^{2}\right)$. The two frames shared one element location at the center of the monitor, while containing two other elements located at horizontally opposite positions relative to the center (see Figure 1A). Each frame was presented for $30 \mathrm{~ms}$; the interstimulus interval $\left(\mathrm{ISI}_{\mathrm{V}}\right.$ ) between the two frames was randomly selected from the range of 50-230 ms, with a step size of $30 \mathrm{~ms}$.

Mono sound beeps $(1,000 \mathrm{~Hz}$ pure tone, $65 \mathrm{~dB} \mathrm{SPL}, 30 \mathrm{~ms}$, except in Experiment 5 where pure tones with pitches of either $1,000 \mathrm{~Hz}$ or $500 \mathrm{~Hz}$ were given) were generated and delivered via an M-Audio card (Delta 1010) to a headset (Philips, SHM1900). No ramps were applied to modulate the shape of the tone envelope. To ensure accurate timing of the auditory and visual stimuli, the duration of the visual stimuli and the synchronization of the auditory and visual stimuli were controlled via the monitor's vertical synchronization pulses. The experimental program was written with Matlab (Mathworks Inc.) and the Psychophysics Toolbox (Brainard, 1997; Kleiner et al., 2007).

\section{Experimental Design \\ Practice}

Prior to the formal experiment, participants were familiarized with Ternus displays of either typical "element motion" (with an interval of $50 \mathrm{~ms}$ ) or "group motion" (with an interval of $260 \mathrm{~ms}$ ) in a practice block. They were asked to discriminate the two types of apparent motion by pressing the left or the right mouse button, respectively. The mapping between response button and type of motion was counterbalanced across participants. During practice, when an incorrect response was made, immediate feedback appeared on the screen showing the correct response (i.e., element or group motion). The practice session continued until the participant reached a mean accuracy of 95\%. All participants achieved this within 120 trials.

\section{Pre-test}

For each participant, the transition threshold between element and group motion was determined in a pre-test session. A trial began with the presentation of a central fixation cross lasting 300 to $500 \mathrm{~ms}$. After a blank screen of $600 \mathrm{~ms}$, the two Ternus frames were presented, synchronized with two auditory tones [i.e., baseline: $\operatorname{ISIV}$ (isual) = ISIA(uditory)]; this was followed by a blank screen of 300 to $500 \mathrm{~ms}$, prior to a screen with a question mark prompting the participant to make a two-alternative forced-choice response indicating the type of perceived motion (element or group motion). The $\mathrm{ISI}_{\mathrm{V}}$ between the two visual frames was randomly selected from one of the following seven intervals: $50,80,110,140,170,200$, and $230 \mathrm{~ms}$. There were 40 trials for each level of ISI $_{\mathrm{V}}$, counterbalanced with left- and rightward apparent motion. The presentation order of the trials was randomized for each participant. Participants performed a total of 280 trials, divided into 4 blocks of 70 trials each. After completing the pre-test, the proportions of the group motion responses across seven intervals were fitted to the psychometric curve using a logistic function (Treutwein and Strasburger, 1999; Wichmann and Hill, 2001). The transitional threshold, that is, the point of subjective equality (PSE) at which the participant was likely to report the two motion percepts equally, was calculated by estimating $50 \%$ of reporting of group motion on the fitted curve. The just noticeable difference (JND), an indicator of the sensitivity of apparent motion discrimination, was calculated as half of the difference between the lower (25\%) and upper (75\%) bounds of the thresholds from the psychometric curve.

\section{Main Experiments}

In the main experiments, the procedure for presenting visual stimuli was the same as in the pre-test session, except that prior to the occurrence of two Ternus-display frames, an 
A
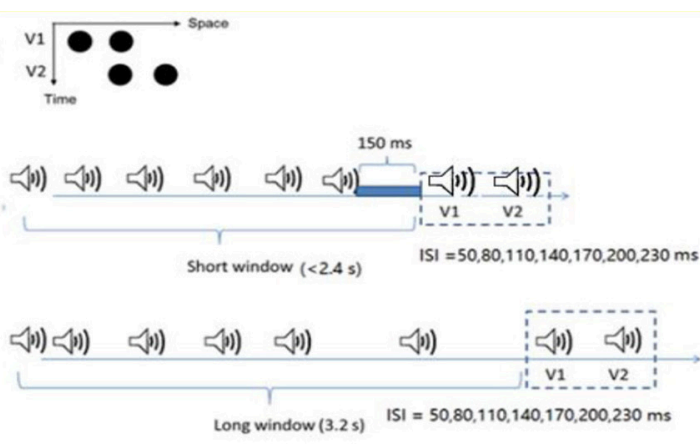

C
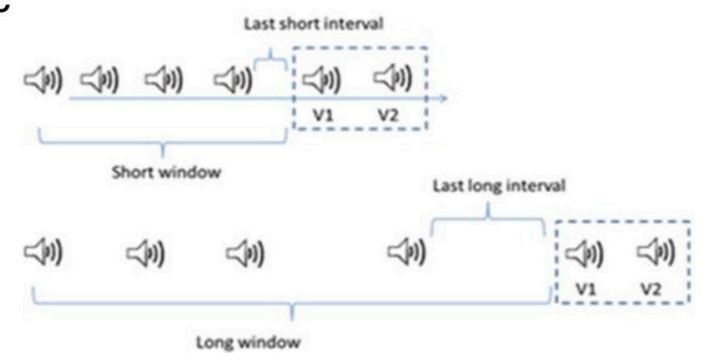

B
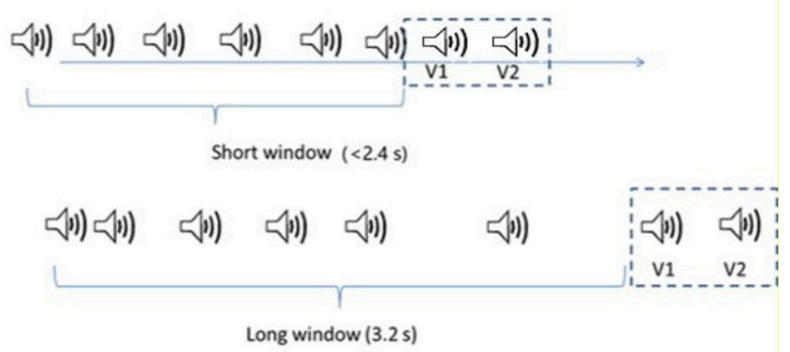

D

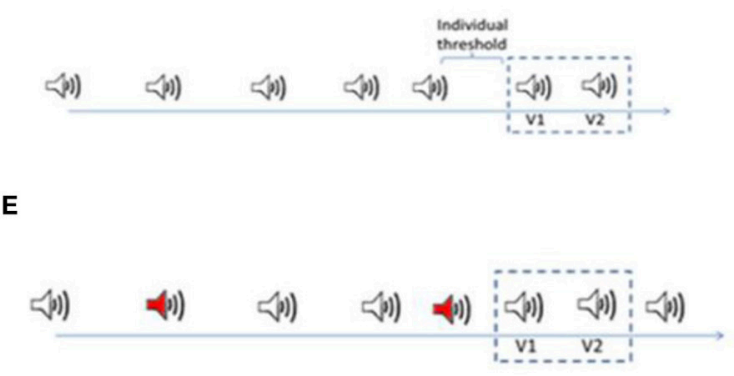

FIGURE 1 | Stimuli configurations for the four experiments. (A) Ternus display: two alternative motion percepts of the Ternus display - "element" motion for the short ISIs, with the middle black dot perceived as remaining static while the outer dots are perceived to move from one side to the other. "Group" motion for long ISIs, with the two dots perceived as moving in tandem. The auditory sequence consisted of 6 to 8 beeps (with 7 beeps as the most frequent cases). The Ternus display, with 50 to $230 \mathrm{~ms}$ interval between the two frames, was followed by a blank interval of $150 \mathrm{~ms}$ to the offset of the last beep in the short time window condition (the total interval length from the onset of the first beep to the onset of the first visual Ternus frame was less than $2.4 \mathrm{~s}$ ), and $3.2 \mathrm{~s}$ in the long time window condition. In both the short and long window conditions, two beeps were synchronously paired with two visual Ternus frames. (B) The configuration was nearly the same as in (A), but for the short window condition, the two frames followed immediately with the last beep. (C) The competition between the mean interval in temporal window and the last auditory interval upon the visual Ternus motion. The mean auditory inter-intervals/last auditory intervals could be longer (transition threshold $+70 \mathrm{~ms}$ ) or shorter (transition threshold -70 ms) than the threshold between the element-and group-motion percept. The lengths for both short and long time windows were the same as in (A). (D) Two types of auditory sequences with five auditory intervals were composed: one with its geometric mean 70 ms shorter than the transition threshold of the visual Ternus motion ("Short" condition), and the other with its geometric mean 70 ms longer than the transitional threshold ("Long" condition). The last auditory interval before the onset of Ternus display was fixed at the individual "transitional threshold" for both sequences. (E) The configuration was similar as in $\mathrm{C}$ but the sound sequence had up to two oddball sounds $(500 \mathrm{~Hz}$, here we showed two oddball sounds with red labels). The remaining regular sounds were of $1,000 \mathrm{~Hz}$ (including the two beeps synchronous with the two visual frames).

auditory sequence consisting a variable number of 6-8 beeps was presented (see below for the details of the onset of Ternus-display frames relative to that of the auditory sequence). A trial began with the presentation of a central fixation marker, randomly for 300 to $500 \mathrm{~ms}$. After a 600-ms blank interval, the auditory train and the visual Ternus frames were presented (see Figure 1A), followed sequentially by a blank screen of 300 to $500 \mathrm{~ms}$ and a screen with a question mark at the screen center prompting participants to indicate the type of motion they had perceived: element vs. group motion (non-speeded response). During the experiment, observers were simply asked to indicate the type of visual motion ("element" or "group" motion) that they perceived, while ignoring the beeps. After the response, the next trial started following a random inter-trial interval of 500 to $700 \mathrm{~ms}$.

In Experiment 1, the visual Ternus frames were preceded by an auditory sequence of $6-8$ beeps with the geometric mean of inter-stimulus interval [ISIA(uditory), i.e., ISI $_{A}$ ], manipulated to be $70 \mathrm{~ms}$ shorter than, or $70 \mathrm{~ms}$ longer than the transition threshold estimated in the pre-test. The [ISIV(isual), i.e., ISIV] between the two visual Ternus frames was randomly selected from one of the following seven intervals: 50, 80, 110, 140, 170, 200 , and $230 \mathrm{~ms}$. The total auditory sequence consisted of 6-8 beeps. Visual Ternus frames were presented on most of all trials (672 trials in total) following the last beep; the remaining were catch trials (72 trials) in which the frames were inset in the sound sequence to break up anticipatory processes. For the short time window of the auditory sequence, the time interval from the onset of the first beep to the onset of the first visual frame was less than $2.4 \mathrm{~s}$, and the gap interval between the offset of the last beep and the onset of the first Ternus frame was $150 \mathrm{~ms}$. For the long time window, the total interval from the onset of the sound to the first visual frame was $3.2 \mathrm{~s}$. In both the short and long window conditions, two beeps were synchronously paired with two visual Ternus frames. All the trials were randomized and organized in 12 blocks ( 62 trials for each block).

In Experiment 2, the settings were the same as in Experiment 1, except for the condition: the visual frames were following immediately with the offset of the last beep.

In Experiment 3, we introduced two factors of interval modulations: the mean interval of temporal window and the last 
auditory interval. The mean auditory inter-intervals and the last auditory intervals could be larger (transition threshold $+70 \mathrm{~ms}$ ) or shorter (transition threshold $-70 \mathrm{~ms}$ ) than the threshold between the element- and group- motion percept. Therefore, there were four combinations of the "interval" conditions: both the mean interval and the last interval were shorter (i.e., "MeanSLastS"); the mean interval was shorter but the last interval was longer ("MeanSLastL"); the mean was longer but the last interval was shorter ("MeanLLastS"); and both the mean interval and the last interval were longer ("MeanLLastL"). The onset of the two visual Ternus frames ( $30 \mathrm{~ms})$ was accompanied by a (30-ms) auditory beep (i.e., $\mathrm{ISI}_{\mathrm{V}}=\mathrm{ISI}_{\mathrm{A}}$ ).

In Experiment 4 we compared two auditory sequences: one with its geometric mean $70 \mathrm{~ms}$ shorter than the transition threshold of the visual Ternus motion (hereafter the "Short" condition), and the other with its geometric mean $70 \mathrm{~ms}$ longer than the transitional threshold (hereafter the "Long" condition). Instead of randomization of the five auditory intervals (excluding the final synchronous auditory interval with the visual Ternus interval), the last auditory interval before the onset of Ternus display was fixed at the "transitional threshold" for both sequences. The rest four intervals were chosen randomly such that the coefficient of variance $(\mathrm{CV})$ of the auditory sequence was in the range between 0.1 and 0.2 , which is the normal range of CV for human observers (Allan, 1974; Getty, 1975; Penney et al., 2000). By this manipulation, we expected to minimize the influence of the potential recency effect caused by the last auditory interval. The audiovisual Ternus frames were appended at the end of these sequences for $85.7 \%$ trials (with 672 trials out of 784 trials), in which the Ternus display appeared at the end of the sound sequence (the "onset" of first visual frame was synchronized with 6 th beep). The remaining were 112 catch trials, in which 56 trials had the Ternus displays at the beginning of the sound sequence (i.e., the "onset" of the first visual frame was synchronized with the second beep), and the rest 56 trials at middle temporal locations (i.e., the "onset" of the first visual frame was synchronized with the 4 th beep). Those catch trials were used to avoid potential anticipatory attending to the visual events appearing at the end of the sound sequence. The total 784 trials were randomized and organized in 14 blocks, with each of 56 trials.

In Experiment 5, we used three types of auditory sequences, in which the mean auditory interval was either shorter than, equal to or longer than the individual transitional threshold of Ternus motion. The auditory sequence consisted of 8 to 10 beeps, including those accompanying the two visual Ternus frames, with the latter being inserted mainly at the 6 th -7 th positions (504 trials), and followed by $0-2$ beeps (number selected at random), to minimize expectations for the onset of the visual Ternus frames. Two of the beeps (the 6 th and the 7 th) were synchronously paired with two visual Ternus frames which were separated by a visual ISI (ISIV) that varied from 50 to $230 \mathrm{~ms}$ (for the critical beeps, $\left.\mathrm{ISI}_{\mathrm{V}}=\mathrm{ISI}_{\mathrm{A}}\right)$. There were up to two oddball tones $(500 \mathrm{~Hz})$ in the sound sequence, while the remaining regular sounds were of $1,000 \mathrm{~Hz}$ (including the two beeps synchronous with the two visual frames). Participants completed a dual-task in which they not only made discriminations of the
Ternus display (“element motion” vs. "group motion”) but also reported the number of oddball sounds (0-2) (Figure 1).

\section{RESULTS}

\section{Experiment 1: The Effect of Short Temporal Window (With a Temporal Gap Between Auditory Sequence and Visual Ternus) vs. Long Temporal Window}

The PSEs for the short window and long window were 149.4 $( \pm 5.6$, standard error) $\mathrm{ms}$ and $141.2( \pm 4.8) \mathrm{ms}$. The main effect of temporal window was significant, $F_{(1,13)}=6.878, p=0.021$, $\eta_{g}^{2}=0.346$. The PSEs for short interval and long interval were $145.5( \pm 5.2) \mathrm{ms}$ and $145.0( \pm 4.8) \mathrm{ms}$, the main effect of mean interval was not significant, $F_{(1,13)}=0.120, p=0.735$, $\eta_{g}^{2}=0.009$. The interaction effect between factors of window and interval was not significant, $F_{(1,13)}=1.033, p=0.328$, $\eta_{g}^{2}=0.074$. For the JNDs, both the main effects of temporal window and mean interval were not significant, $F_{(1,13)}=3.419$, $p=0.087, \eta_{g}^{2}=0.208$ and $F_{(1,13)}=0.089, p=0.770, \eta_{g}^{2}=$ 0.007 . And the interaction effect between the two factors was not significant, $F_{(1,13)}=2.863, p=0.114, \eta_{g}^{2}=0.180($ Figures 2,4$)$.

\section{Experiment 2: The Effect of Short Temporal Window (Without a Gap Between Auditory Sequence and Visual Ternus) vs. Long Temporal Window}

The PSEs for the short window and long window were 168.7 $( \pm 6.2) \mathrm{ms}$ and $156.2( \pm 5.7)$. The PSE for short window was larger

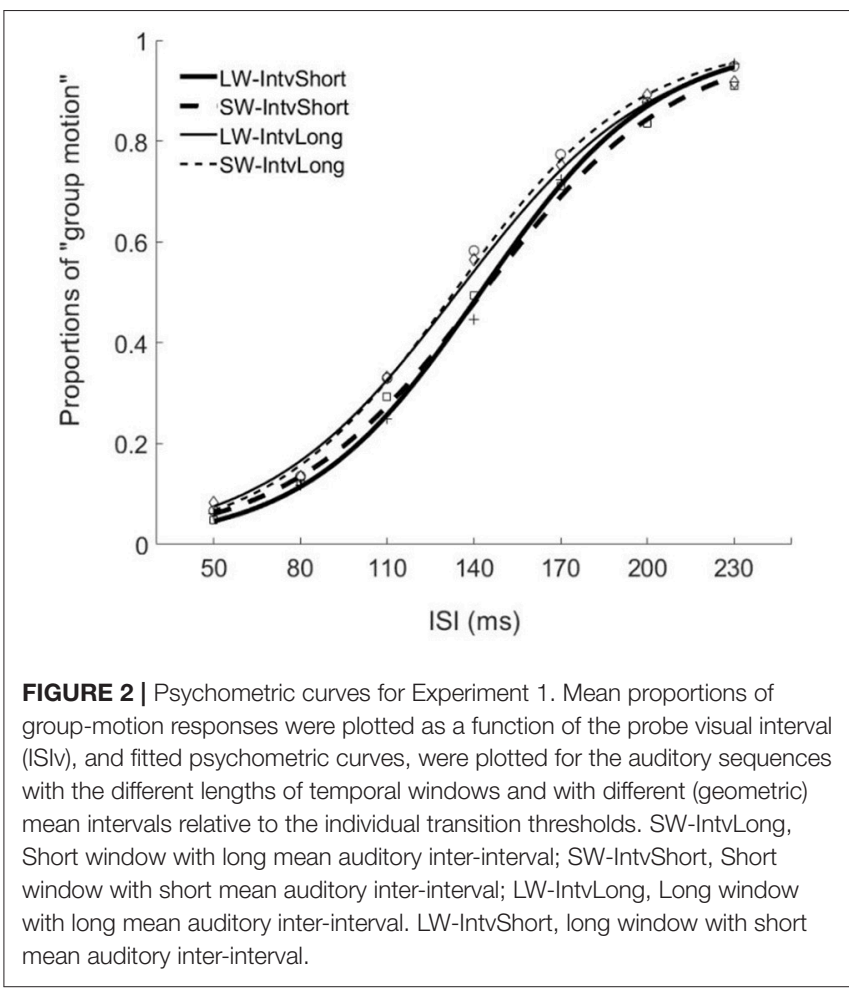


than the one in long window, $F_{(1,12)}=20.860, p=0.001, \eta_{g}^{2}=$ 0.635 . The PSEs for short interval and long interval were 163.8 $( \pm 6.0) \mathrm{ms}$ and $161.0( \pm 5.8)$, the main effect of mean interval

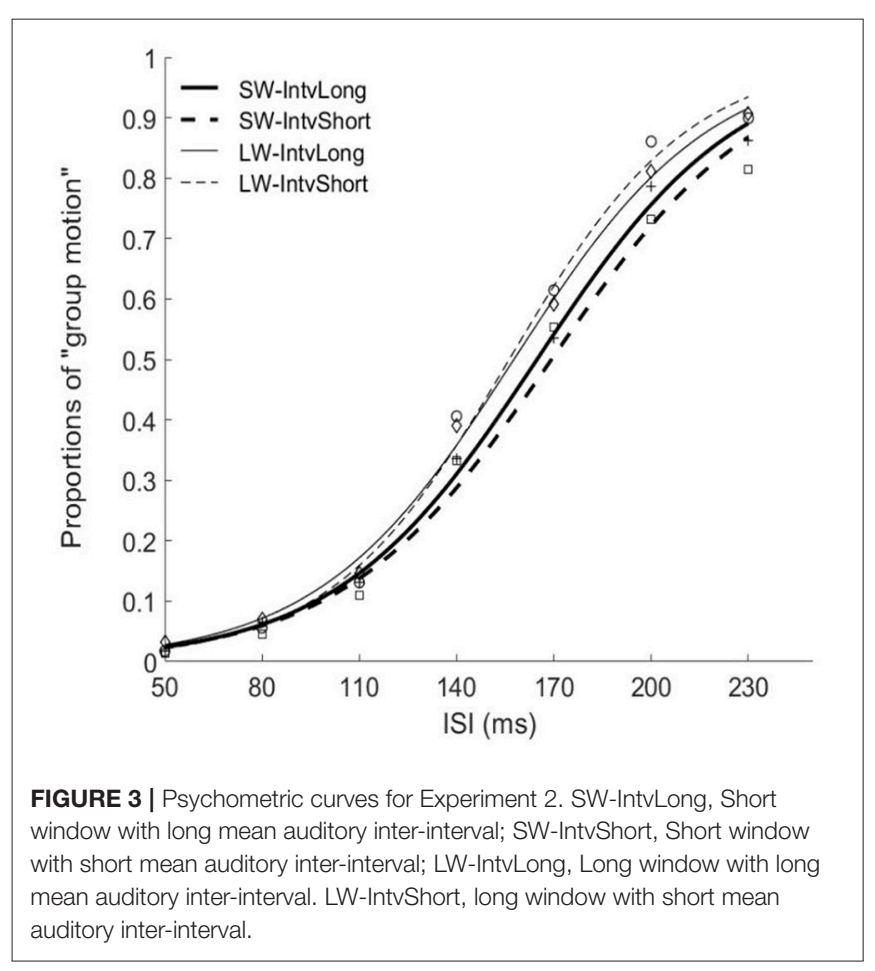

was not significant, $F_{(1,12)}=1.869, p=0.197, \eta_{g}^{2}=0.135$. Importantly, the interaction effect between factors of window and interval was significant, $F_{(1,12)}=5.090, p=0.044, \eta_{g}^{2}=0.298$. Further simple effect analyses showed that for short interval, the PSE in short window $(172.7 \pm 7.3 \mathrm{~ms})$ was larger than the one $(154.9 \pm 5.3 \mathrm{~ms})$ in long window, $p=0.001$. For long interval, the PSE in short window $(164.7 \pm 5.5 \mathrm{~ms})$ was larger than the one $(157.3 \pm 6.4 \mathrm{~ms})$ in long window, $p=0.034$. On the other hand, for the short window, the PSE in short interval (172.7 \pm $7.3 \mathrm{~ms}$ ) was larger than the one in long interval (164.7 $\pm 5.5 \mathrm{~ms})$, $p=0.044$. However, for the long window, the PSEs are equal in both intervals (154.9 vs. $157.3 \mathrm{~ms}$ for short and long intervals), $p=0.377$.

For the JNDs, both the main effects of temporal window and mean interval were not significant $\left[F_{(1,12)}=2.479, p=0.141\right.$, $\eta_{g}^{2}=0.171$ and $\left.F_{(1,12)}=0.282, p=0.605, \eta_{g}^{2}=0.023\right]$. The interaction effect between the two factors was not significant, $F_{(1,12)}=0.408, p=0.535, \eta_{g}^{2}=0.033$ (Figures 3, 4).

\section{Experiment 3: Central Tendency Effect vs. Last Interval}

The PSEs for the short mean interval and long mean interval were $143.2( \pm 7.4) \mathrm{ms}$ and $135.3( \pm 9.5)$. The main effect of mean interval was significant, $F_{(1,6)}=9.070, p=0.024, \eta_{g}^{2}=0.602$. The PSEs for short last interval and long last interval were 155.8 $( \pm 9.7) \mathrm{ms}$ and $122.6( \pm 7.5) \mathrm{ms}$, respectively. The main effect of last interval was significant, $F_{(1,6)}=65.970, p=0.000, \eta_{g}^{2}=$ 0.917 . The interaction effect between factors of mean interval

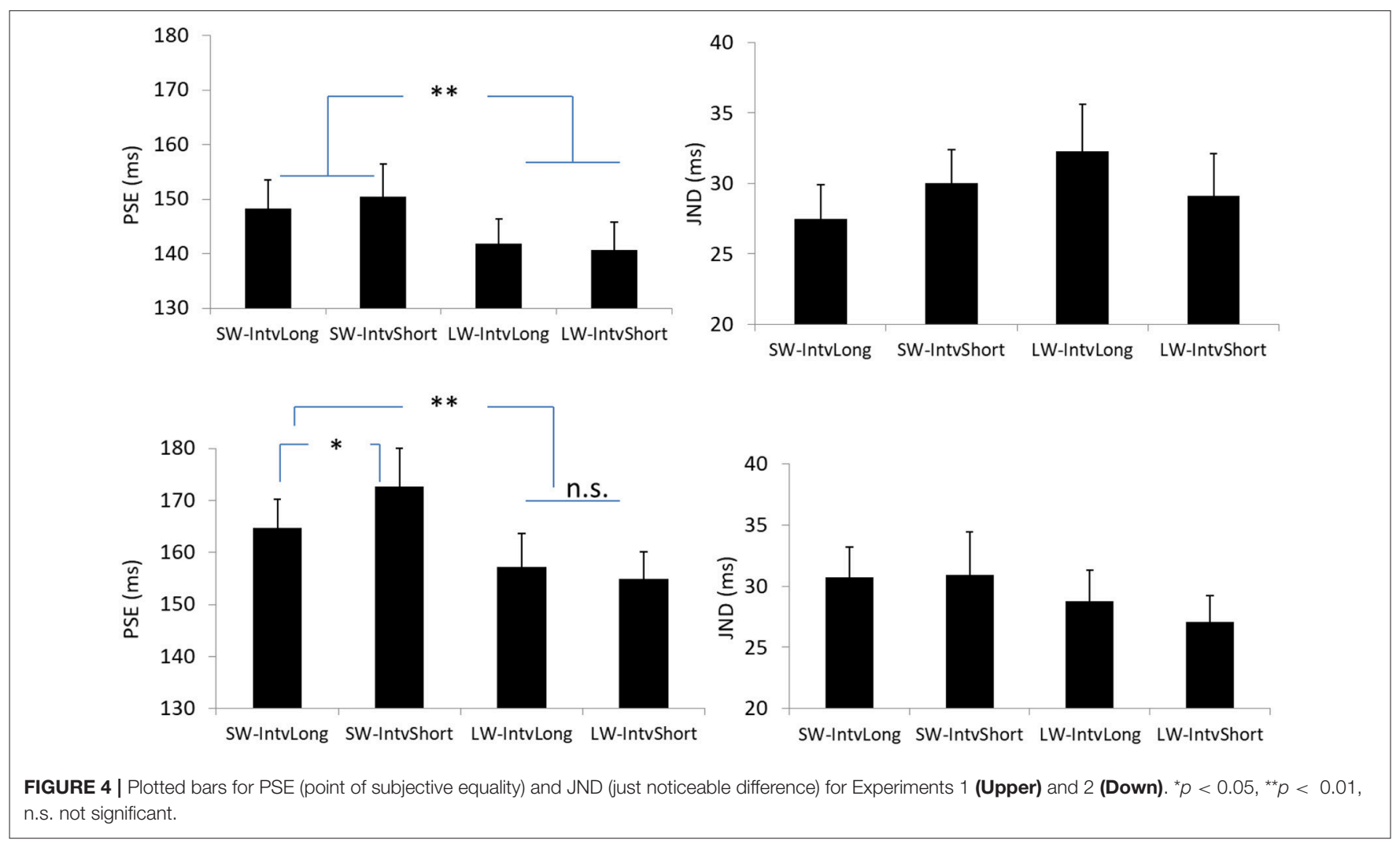


and last interval was not significant, $F_{(1,6)}=0.195, p=0.674$, $\eta_{g}^{2}=0.031$. For the JNDs, the JND in short last interval (24.8 $\pm 1.3 \mathrm{~ms})$ was larger than the one in long last interval $(21.5 \pm$ $1.6 \mathrm{~ms}), F_{(1,6)}=11.590, p=0.014, \eta_{g}^{2}=0.659$. However, the main effect of mean interval was not significant, $F_{(1,6)}=0.762$, $p=0.416, \eta_{g}^{2}=0.113$. The interaction effect between the two factors was also not significant, $F_{(1,6)}=0.109, p=0.753, \eta_{g}^{2}=$ 0.018. (Figures 5, 6).

\section{Experiment 4: Central Tendency Effect but With the Last Interval Fixed}

Here we made formal manipulation by keeping the last interval fixed for the "Short" and "Long" auditory sequences. Figure 7 depicts the responses from a typical participant. The PSEs were $153.1( \pm 7.3), 137.9( \pm 9.1)$ for the "Short" and "Long" conditions, $t_{(11)}=3.640, p<0.01$. Participants perceived more dominant percept of Element motion in the "Short" condition than in the "Long" condition, consistent with the findings of the previous experiments. That is, the auditory ensemble mean still assimilated visual Ternus apparent motion when the last interval of the auditory sequence was fixed. Therefore, the audiovisual interactions we found were unlikely only due to the recency effect.

\section{Experiment 5: Central Tendency Effect With Attentional Modulation}

The PSEs for the baseline, short, equal, and long intervals were 135.9( \pm 3.3$), 171.1( \pm 8.9), 151.5( \pm 9.5)$, and142.1( \pm 7.4$) \mathrm{ms}$, the main effect of mean interval was significant, $F_{(2,39)}=9.020, p<$ $0.001, \eta_{g}^{2}=0.410$. Bonferroni corrected comparison showed that the PSE for baseline was smaller than the one in short condition, $p=0.014$. PSE for short interval condition was larger than the one in equal condition, $p=0.01$; and the PSE for short interval was also larger than the ones in the equal and long intervals, $p=0.019$ and $p=0.010$. However, the PSEs were equal for both

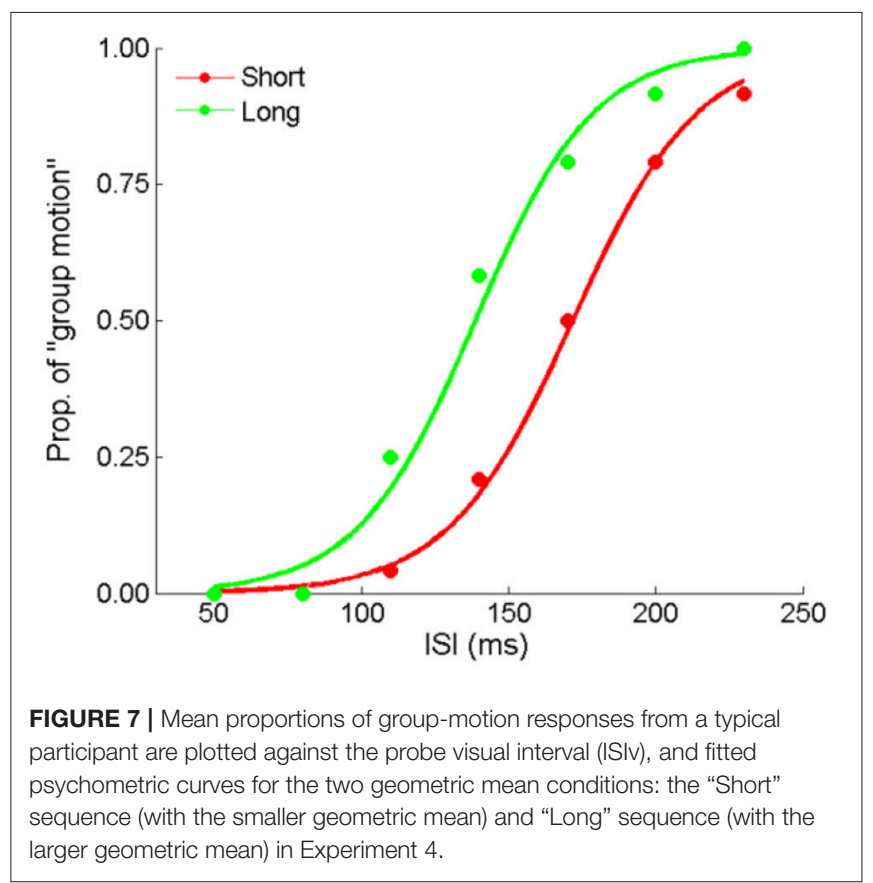

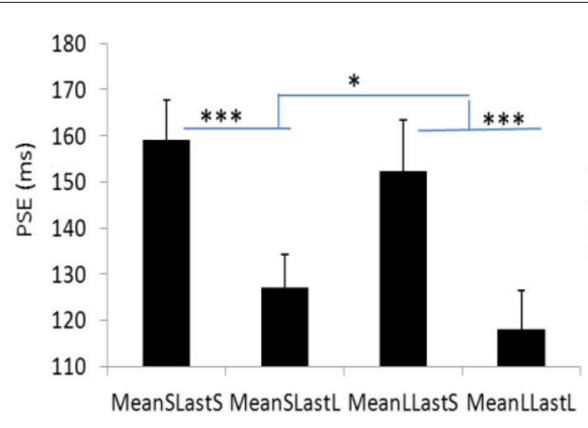

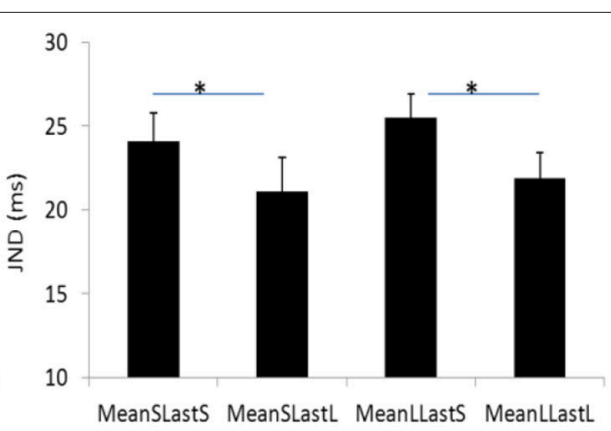

FIGURE 6 | Plotted bars for PSE (point of subjective equality) and JND (just noticeable difference) for Experiment $3 .{ }^{*} p<0.05,{ }^{* *} p<0.01$, and ${ }^{* \star *} p<0.001$. 
"equal" and "long" conditions, $p=0.411$. The PSEs were equal for both "baseline" and "equal" condition, $p=0.603$, and were equal between "baseline" and "long" conditions, $p=1$.

The JNDs for the baseline, short, equal, and long intervals were $32.2( \pm 3.7), 39.3( \pm 5.1), 44.9( \pm 7.0)$, and $40.0( \pm 4.4) \mathrm{ms}$, respectively. The main effect of mean interval was not significant, $F_{(3,39)}=2.741, p=0.056, \eta_{g}^{2}=0.174$ (Figures 8, 9).

The mean correct rate for reporting the number of oddball sounds was $83.0 \pm 3.1 \%$, one sample $T$-test with comparison of $50 \%$ showed the correct rate was above the chance level $\left[t_{(13)}=10.518, p=9.984 \times 10^{-8}\right]$.

\section{DISCUSSION}

Central tendency, the tendency of judgments of quantitative properties (lengths, durations etc) for given stimuli to gravitate toward their mean, is one of the most robust perceptual effects. The present study has shown that perceptual averaging of temporal property-auditory intervals, assimilates the visual interval between the two Ternus-display frames, and biases the perception of Ternus apparent motion (either to be dominant "element motion" or dominant "group motion"). This finding is consistent with the large body of literature on temporal-context and central tendency effects, within the broader framework of Bayesian optimization (Jazayeri and Shadlen, 2010; Shi et al., 2013; Roach et al., 2017), whereby incorporating the mean of the statistical distribution in the estimation would assimilate the estimates toward the mean-known as "central tendency effect" (Jazayeri and Shadlen, 2010; Burr et al., 2013; Karaminis et al., 2016).

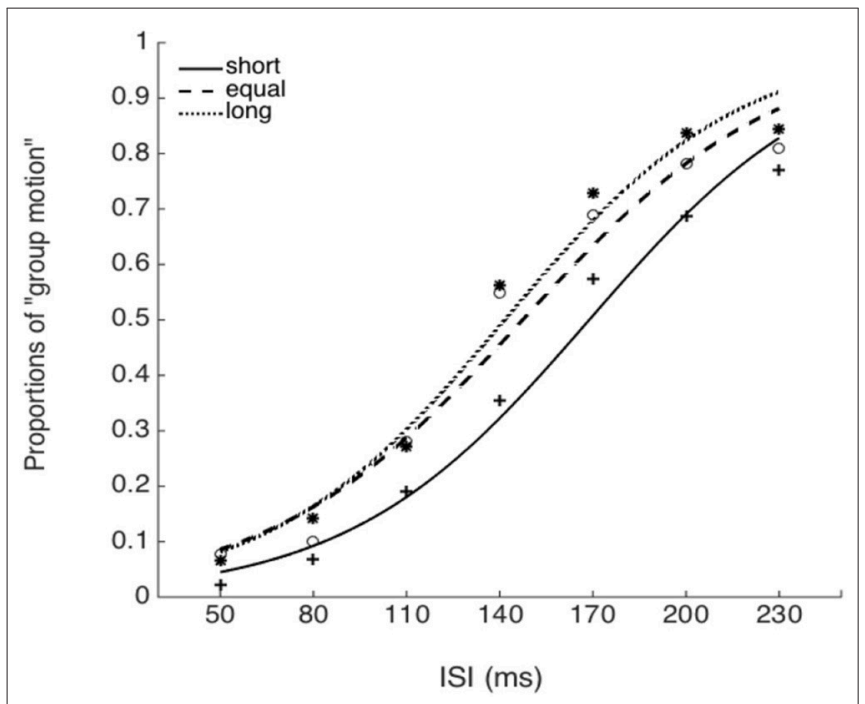

FIGURE 8 | Psychometric curves for Experiment 5. Short (solid line), the mean auditory inter-interval is shorter than the PSE for visual Ternus motion; Equal (dashed line), the mean auditory inter-interval is equal to the PSE for visual Ternus motion; Long (dotted line), the mean auditory inter-interval is longer than the PSE for visual Ternus motion. The PSE ("transitional threshold") of Ternus motion was established by a pre-test for each individual.
By using the paradigm of temporal ventriloquism and the probe of visual Ternus display (Chen et al., 2010; Shi et al., 2010; Chen and Vroomen, 2013), we have previously shown that the auditory capture effect upon the visual events, in which the perceived visual interval was biased by concurrently presented auditory events. Observers tended to report the illusory visual (apparent motion) percepts with the concurrent presence of auditory beeps. However, the visual-auditory integration effect is subject to the temporal reference, i.e., the time interval between the critical visual probe and the sound sequence, the mean auditory interval and the critical interval between the last auditory stimulus and the onset of visual events. In our current setting, when the total time interval between the onset of auditory signal and the onset of visual events was above $3 \mathrm{~s}(3.2 \mathrm{~s})$, it gave rise to a diminished central tendency effect. On the contrary, when this time interval was less than $2.4 \mathrm{~s}$, the shortened time reference increased the likelihood of central tendency effect-materialized in the effect of "geometric" perceptual averaging for auditory intervals upon the visual Ternus motion. These findings indicate a general temporal framework of crossmodal integration. As stated in a theoretical construct of temporal perception, known as the "subjective present" - a mechanism of temporal integration binds successive events into perceptual units of $3 \mathrm{~s}$ duration (Pöppel, 1997). Such a temporal integration, which is automatic and pre-semantic, is also operative in movement control and other cognitive activities. In this hierarchical temporal model, the temporal reference for temporal binding could be extended but limited within $3 \mathrm{~s}$, together with a memory store (Pöppel, 1997; Pöppel and Bao, 2014). When the framework exceeds $3 \mathrm{~s}$, the integration of the preceding auditory interval information could be decayed, which hence makes the auditory assimilation effect reduced.

Interestingly, even with the presumed short temporal window (within $2.4 \mathrm{~s}$ ), by inserting a short temporal gap $(150 \mathrm{~ms})$ between the offset of the very last beep and the onset of the first visual frame, we found the central tendency effect was reduced, and the effect was similar to the results in long temporal window condition ( $3.2 \mathrm{~s})$. This finding suggests that the "imminent" and most recent ("immediate") temporal gap before the target visual event is critical for the development of the central tendency effect. This inference is further substantiated by the results from Experiments 2 and 3. In Experiment 2, with the configuration of "short window," we eliminated the short gap $(150 \mathrm{~ms})$ between the offset of the last beep and the onset of the visual frames. We found that the central tendency effect (short mean interval. vs. long mean interval) reappeared, though it still remains absent in the condition of "long window." Moreover, in Experiment 3, we further found that the assimilation effect of the last interval dominates that of the mean auditory interval. This indicates that the last auditory interval wins the competition over the mean interval in driving the crossmodal assimilation.

However, the central tendency effect was less dependent on attentional modulation. Using the dual-tasks of reporting the percept of visual Ternus motion and the number of oddball stimuli [i.e., identifying the number of $500 \mathrm{~Hz}$ beep(s) within a sound sequence], we again found the central tendency effect was robust. The observers have invested large attentional resources to 

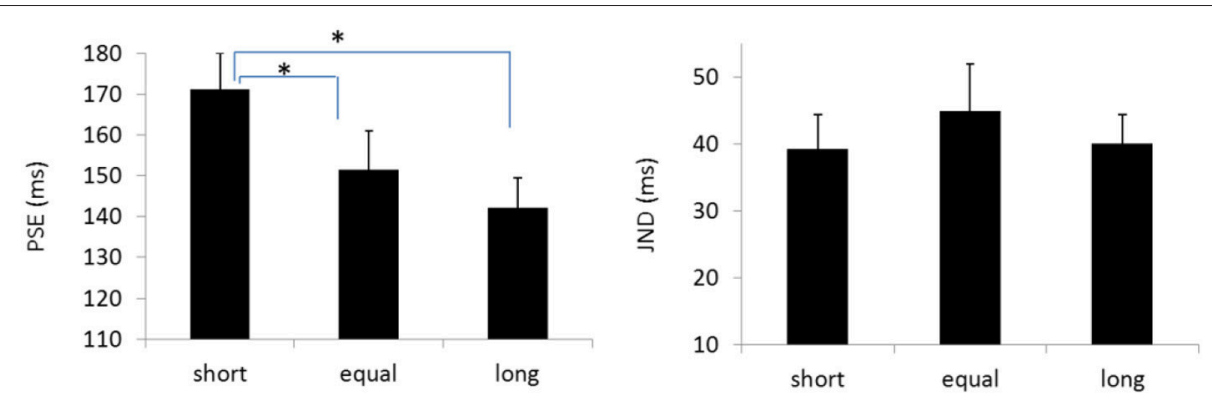

FIGURE 9 | Plotted bars for PSE (point of subjective equality) and JND (just noticeable difference) for Experiments 5 , ${ }^{*} p<0.05$.

obtain the decent performance of counting the oddball sounds. Nevertheless, the performance of crossmodal assimilation effect still survived. Therefore, the central tendency effect as shown in the present study, has demonstrated its automatic and attentional-less demanding nature during crossmodal interaction (Vroomen et al., 2001; Wahn and Konig, 2015).

The current study has some limitations. Indeed, the temporal reference before the target visual Ternus display includes intervals composed by stimuli with different configurations. The auditory sequence was organized by filled-durations with multiple beeps, and there was a transition of intra-modal perceptual grouping (with sounds) to cross-modal grouping when the last beep was followed by the onset of the first visual Ternus frame (with audiovisual events) (Burr et al., 2013). However, the "critical" time window for multisensory integration was presented as an "empty interval" between the two visual frames. Therefore, the visual probe we adopted in current experimental paradigm might restrict the manifestation of assimilation effect, which was probably due to the differential timing sensitivities to the "filled-duration" in auditory sequence vs. "empty-duration" in the visual probe (Rammsayer and Lima, 1991; Grondin, 1993; Rammsayer, 2010). Moreover, the temporal window, as shown in the auditory sequence, covaried with the mean ISIs (mean auditory intervals). This potential confound remains even although we have manipulated the comparisons of durations between the mean ISIs and the critical interval between the two visual frames (Experiments 1, 2, 3, and 5), and tried to tease apart the "central tendency effect" vs. "recency effect" by fixing the last intervals. Further research is needed to elucidate this point.

Taken together, the current study has shown that crossmodal assimilation in temporal domain is shaped by the temporal reference, in which the observers use the temporal information by dynamically averaging the intervals (as they unfold in time sequence) and exploiting the last interval before the target events. The central tendency effect in temporal domain, similar to the central effect associated with other sensory properties such as weights and hues, is adaptively subject to the frame of reference (Hollingworth, 1910; Helson, 1947, 1948; Helson and Himelstein, 1955; Sherif et al., 1958; Thomas and Jones, 1962; Helson and Avant, 1967; Thomas et al., 1973; Hébert et al., 1974; Thomas and Strub, 1974; Newlin et al., 1978; Burr et al., 2013; Karaminis et al., 2016). Importantly, the temporal information near the target event is critical for crossmodal assimilation, wherein the recency effect prevails over the central tendency effect during the assimilation process (Burr et al., 2013; Karaminis et al., 2016). Crossmodal assimilation is more dependent on the temporal duration which entails the integration of task-relevant (temporal) information to be efficient within a short window (3s) in addition to efficient working memory functions (Pöppel, 1997; Block and Gruber, 2014; Pöppel and Bao, 2014). However, the crossmodal assimilation is less subject to another process-attentional modulation (Talsma et al., 2010).

\section{AUTHOR CONTRIBUTIONS}

YW conducted Experiment 1 and analyzed data. LC conducted Experiments 2-4, analyzed data and wrote the manuscript.

\section{ACKNOWLEDGMENTS}

This work is funded by the Natural Science Foundation of China (NSFC61527804, 81371206) and was partially funded by NSFC and the German Research Foundation (DFG) in Project Crossmodal Learning, NSFC 61621136008 / DFG TRR-169.

\section{SUPPLEMENTARY MATERIAL}

The Supplementary Material for this article can be found online at: https://www.frontiersin.org/articles/10.3389/fncom. 2018.00039/full\#supplementary-material

Supplement 1: Demo of Ternus Display. 


\section{REFERENCES}

Acerbi, L., Wolpert, D. M., and Vijayakumar, S. (2012). Internal representations of temporal statistics and feedback calibrate motor-sensory interval timing. PLoS Comput. Biol. 8:e1002771. doi: 10.1371/journal.pcbi.1002771

Allan, L. G. (1974). Psychophysical theories of duration discrimination. Percept. Psychophys. 16, 26-34. doi: 10.3758/BF03203244

Alsius, A., Mottonen, R., Sams, M. E., Soto-Faraco, S., and Tiippana, K. (2014). Effect of attentional load on audiovisual speech perception: evidence from ERPs. Front. Psychol. 5:727. doi: 10.3389/fpsyg.2014.00727

Alsius, A., Navarra, J., Campbell, R., and Soto-Faraco, S. (2005). Audiovisual integration of speech falters under high attention demands. Curr. Biol. 15, 839-843. doi: 10.1016/j.cub.2005.03.046

Block, R. A., and Gruber, R. P. (2014). Time perception, attention, and memory: a selective review. Acta Psychol. 149, 129-133. doi: 10.1016/j.actpsy.2013.11.003

Brainard, D. H. (1997). The psychophysics toolbox. Spat. Vis. 10, 433-436. doi: $10.1163 / 156856897 \mathrm{X} 00357$

Bruns, P., and Röder, B. (2015). Sensory recalibration integrates information from the immediate and the cumulative past. Sci. Rep. 5:12739. doi: $10.1038 /$ srep 12739

Buchan, J. N., and Munhall, K. G. (2011). The influence of selective attention to auditory and visual speech on the integration of audiovisual speech information. Perception 40, 1164-1182. doi: 10.1068/p6939

Burr, D., Della Rocca, E., and Morrone, M. C. (2013). Contextual effects in intervalduration judgements in vision, audition and touch. Exp. Brain Res. 230, 87-98. doi: $10.1007 / \mathrm{s} 00221-013-3632-\mathrm{z}$

Cardinal, L. J. (2015). Central tendency and variability in biological systems: part 2. J. Commun. Hosp. Intern. Med. Perspect. 5:28972. doi: 10.3402/jchimp.v5.28972

Chen, L., Shi, Z., and Muller, H. J. (2010). Influences of intra- and crossmodal grouping on visual and tactile Ternus apparent motion. Brain Res. 1354, 152-162. doi: 10.1016/j.brainres.2010.07.064

Chen, L., and Vroomen, J. (2013). Intersensory binding across space and time: a tutorial review. Atten. Percept. Psychophys. 75, 790-811. doi: 10.3758/s13414-013-0475-4

Chen, L., Zhang, M., Ai, F., Xie, W., and Meng, X. (2016). Crossmodal synesthetic congruency improves visual timing in dyslexic children. Res. Dev. Disabil. 55, 14-26. doi: 10.1016/j.ridd.2016.03.010

Chen, Y. C., and Spence, C. (2017). Assessing the role of the 'unity assumption' on multisensory integration: a review. Front. Psychol. 8:445. doi: 10.3389/fpsyg.2017.00445

Chuen, L., and Schutz, M. (2016). The unity assumption facilitates crossmodal binding of musical, non-speech stimuli: the role of spectral and amplitude envelope cues. Atten. Percept. Psychophys. 78, 1512-1528. doi: 10.3758/s13414-016-1088-5

Cohen, M. A., Dennett, D. C., and Kanwisher, N. (2016). What is the bandwidth of perceptual experience? Trends Cogn. Sci. 20, 324-335. doi: 10.1016/j.tics.2016.03.006

Cowan, N. (2001). Metatheory of storage capacity limits. Behav. Brain Sci. 24, 154-176. doi: 10.1017/S0140525X0161392X

Donohue, S. E., Green, J. J., and Woldorff, M. G. (2015). The effects of attention on the temporal integration of multisensory stimuli. Front. Integr. Neurosci. 9:32. doi: $10.3389 /$ fnint. 2015.00032

Donohue, S. E., Roberts, K. C., Grent-'t-Jong, T., and Woldorff, M. G. (2011). The cross-modal spread of attention reveals differential constraints for the temporal and spatial linking of visual and auditory stimulus events. J. Neurosci. 31, 7982-7990. doi: 10.1523/JNEUROSCI.5298-10.2011

Duncan, J., Martens, S., and Ward, R. (1997). Restricted attentional capacity within but not between sensory modalities. Nature 387, 808-810.

Ernst, M., and Di Luca, M. (2011). "Multisensory perception: from integration to remapping," in Sensory Cue Integration, ed J. Trommershäuser (New York, NY: Oxford University Press), 225-250.

Friston, K. (2010). The free-energy principle: a unified brain theory? Nat. Rev. Neurosci. 11, 127-138. doi: 10.1038/nrn2787

Getty, D. J. (1975). Discrimination of short temporal intervals: a comparison of two models. Percept. Psychophys. 18, 1-8. doi: 10.3758/BF03199358

Grondin, S. (1993). Duration discrimination of empty and filled intervals marked by auditory and visual signals. Percept. Psychophys. 54, 383-394. doi: 10.3758/BF03205274
Gupta, D. S., and Chen, L. (2016). Brain oscillations in perception, timing and action. Curr. Opin. Behav. Sci. 8, 161-166. doi: 10.1016/j.cobeha.2016.02.021

Habets, B., Bruns, P., and Roder, B. (2017). Experience with crossmodal statistics reduces the sensitivity for audio-visual temporal asynchrony. Sci. Rep. 7:1486. doi: 10.1038/s41598-017-01252-y

Hébert, J. A., Bullock, M., Levitt, L., Woodward, K. G., and Mcguirk, F. D. (1974). Context and frequency effects in generalization of a human voluntary response. J. Exp. Psychol. 102, 456-462. doi: 10.1037/h0035877

Helson, H. (1947). Adaptation-level as frame of reference for prediction of psychophysical data. Am. J. Psychol. 60, 1-29. doi: 10.2307/1417326

Helson, H. (1948). Adaptation-level as a basis for a quantitative theory of frames of reference. Psychol. Rev. 55, 297-313. doi: 10.1037/h0056721

Helson, H., and Avant, L. L. (1967). Stimulus generalization as a function of contextual stimuli. J. Exp. Psychol. 73, 565-567. doi: 10.1037/h0024319

Helson, H., and Himelstein, P. (1955). A short method for calculating the adaptation-level for absolute and comparative rating judgments. Am. J. Psychol. 68, 631-637. doi: 10.2307/1418791

Hillock, A. R., Powers, A. R., and Wallace, M. T. (2011). Binding of sights and sounds: age-related changes in multisensory temporal processing. Neuropsychologia 49, 461-467. doi: 10.1016/j.neuropsychologia.2010.11.041

Hillock-Dunn, A., Grantham, D. W., and Wallace, M. T. (2016). The temporal binding window for audiovisual speech: children are like little adults. Neuropsychologia 88, 74-82. doi: 10.1016/j.neuropsychologia.2016.02.017

Hollingworth, H. L. (1910). The central tendency of judgment. J. Philos. Psychol. Sci. Methods 7, 461-469. doi: 10.2307/2012819

Jazayeri, M., and Shadlen, M. N. (2010). Temporal context calibrates interval timing. Nat. Neurosci. 13, 1020-1026. doi: 10.1038/nn.2590

Jones, M. R., and McAuley, J. D. (2005). Time judgments in global temporal contexts. Percept. Psychophys. 67, 398-417. doi: 10.3758/BF03193320

Karaminis, T., Cicchini, G. M., Neil, L., Cappagli, G., Aagten-Murphy, D., Burr, D., et al. (2016). Central tendency effects in time interval reproduction in autism. Sci. Rep. 6:28570. doi: 10.1038/srep28570

Keetels, M., and Vroomen, J. (2008). Tactile-visual temporal ventriloquism: no effect of spatial disparity. Percept. Psychophys. 70, 765-771. doi: 10.3758/PP.70.5.765

Kleiner, M., Brainard, D., Pelli, D., Ingling, A., Murray, R., and Broussard, C. (2007). What's new in psychtoolbox-3. Perception 36, 1-16. doi: $10.1068 / \mathrm{v} 070821$

Klemen, J., Buchel, C., and Rose, M. (2009). Perceptual load interacts with stimulus processing across sensory modalities. Eur. J. Neurosci. 29, 2426-2434. doi: 10.1111/j.1460-9568.2009.06774.x

Klemen, J., and Chambers, C. D. (2012). Current perspectives and methods in studying neural mechanisms of multisensory interactions. Neurosci. Biobehav. Rev. 36, 111-133. doi: 10.1016/j.neubiorev.2011.04.015

Lewkowicz, D. J., and Flom, R. (2014). The audiovisual temporal binding window narrows in early childhood. Child Dev. 85, 685-694. doi: 10.1111/cdev. 12142

Li, Y., Long, J., Huang, B., Yu, T., Wu, W., Li, P., et al. (2016). Selective audiovisual semantic integration enabled by feature-selective attention. Sci. Rep. 6:18914. doi: 10.1038/srep18914

Mégevand, P., Molholm, S., Nayak, A., and Foxe, J. J. (2013). Recalibration of the multisensory temporal window of integration results from changing task demands. PLoS ONE 8:e71608. doi: 10.1371/journal.pone.0071608

Meredith, M. A., Nemitz, J. W., and Stein, B. E. (1987). Determinants of multisensory integration in superior colliculus neurons. I. Temporal Factors J. Neurosci. 7, 3215-3229.

Miller, J. E., Carlson, L. A., and McAuley, J. D. (2013). When what you hear influences when you see: listening to an auditory rhythm influences the temporal allocation of visual attention. Psychol. Sci. 24, 11-18. doi: 10.1177/0956797612446707

Misceo, G. F., and Taylor, N. J. (2011). A "unity assumption" does not promote intersensory integration. Exp. Psychol. 58, 385-390. doi: 10.1027/1618-3169/a000107

Nakajima, Y., ten Hoopen, G., Hilkhuysen, G., and Sasaki, T. (1992). Timeshrinking: a discontinuity in the perception of auditory temporal patterns. Percept. Psychophys. 51, 504-507. doi: 10.3758/BF03211646

Newlin, R. J., Rodgers, J. P., Dickson, J. F., Strub, H., and Thomas, D. R. (1978). The central tendency effect in stimulus generalization: effects of establishing 
a "preexperimental" frome of reference. Percept. Psychophys. 24, 161-167. doi: 10.3758/BF03199543

Parise, C. V., Spence, C., and Ernst, M. O. (2012). When correlation implies causation in multisensory integration. Curr. Biol. 22, 46-49. doi: $10.1016 /$ j.cub.2011.11.039

Penney, T. B., Gibbon, J., and Meck, W. H. (2000). Differential effects of auditory and visual signals on clock speed and temporal memory. J. Exp. Psychol. Hum. Percept. Perform. 26, 1770-1787. doi: 10.1037/0096-1523.26.6.1770

Pöppel, E. (1997). A hierarchical model of temporal perception. Trends Cogn. Sci. 1, 56-61. doi: 10.1016/S1364-6613(97)01008-5

Pöppel, E., and Bao, Y. (2014). "Temporal windows as a bridge from objective to subjective time," in Subjective Time, eds D. Lloyd and V. Arstila (Cambridge, MA: MIT Press), 241-261.

Powers, A. R., Hevey, M. A., and Wallace, M. T. (2012). Neural correlates of multisensory perceptual learning. J. Neurosci. 32, 6263-6274. doi: 10.1523/JNEUROSCI.6138-11.2012

Powers, A. R., Hillock, A. R., and Wallace, M. T. (2009). Perceptual training narrows the temporal window of multisensory binding. J. Neurosci. 29, 12265-12274. doi: 10.1523/JNEUROSCI.3501-09.2009

Rammsayer, T. H. (2010). Differences in duration discrimination of filled and empty auditory intervals as a function of base duration. Atten. Percept. Psychophys. 72, 1591-1600. doi: 10.3758/APP.72. 6.1591

Rammsayer, T. H., and Lima, S. D. (1991). Duration discrimination of filled and empty auditory intervals: cognitive and perceptual factors. Percept. Psychophys. 50, 565-574. doi: 10.3758/BF03207541

Roach, N. W., McGraw, P. V., Whitaker, D. J., and Heron, J. (2017). Generalization of prior information for rapid Bayesian time estimation. Proc. Natl. Acad. Sci. U.S.A. 114, 412-417. doi: 10.1073/pnas.1610706114

Sherif, M., Taub, D., and Hovland, C. I. (1958). Assimilation and contrast effects of anchoring stimuli on judgments. J. Exp. Psychol. 55, 150-155. doi: $10.1037 / \mathrm{h} 0048784$

Shi, Z., and Burr, D. (2016). Predictive coding of multisensory timing. Curr. Opin. Behav. Sci. 8, 200-206. doi: 10.1016/j.cobeha.2016.02.014

Shi, Z., Chen, L., and Muller, H. J. (2010). Auditory temporal modulation of the visual Ternus effect: the influence of time interval. Exp. Brain Res. 203, 723-735. doi: $10.1007 / \mathrm{s} 00221-010-2286-3$

Shi, Z., Church, R. M., and Meck, W. H. (2013). Bayesian optimization of time perception. Trends Cogn. Sci. 17, 556-564. doi: 10.1016/j.tics.2013.09.009

Stevenson, R. A., Siemann, J. K., Schneider, B. C., Eberly, H. E., Woynaroski, T. G., Camarata, S. M., et al. (2014). Multisensory temporal integration in autism spectrum disorders. J. Neurosci. 34, 691-697. doi: 10.1523/JNEUROSCI.3615-13.2014

Stevenson, R. A., Zemtsov, R. K., and Wallace, M. T. (2012). Individual differences in the multisensory temporal binding window predict susceptibility to audiovisual illusions. J. Exp. Psychol. Hum. Percept. Perform. 38, 1517-1529. doi: $10.1037 / \mathrm{a} 0027339$

Sugano, Y., Keetels, M., and Vroomen, J. (2010). Adaptation to motor-visual and motor-auditory temporal lags transfer across modalities. Exp. Brain Res. 201, 393-399. doi: 10.1007/s00221-009-2047-3

Sugano, Y., Keetels, M., and Vroomen, J. (2012). The Build-up and transfer of sensorimotor temporal recalibration measured via a synchronization task. Front. Psychol. 3:246. doi: 10.3389/fpsyg.2012.00246

Sugano, Y., Keetels, M., and Vroomen, J. (2016). Auditory dominance in motor-sensory temporal recalibration. Exp. Brain Res. 234, 1249-1262. doi: 10.1007/s00221-015-4497-0
Talsma, D., Doty, T. J., and Woldorff, M. G. (2007). Selective attention and audiovisual integration: is attending to both modalities a prerequisite for early integration? Cereb. Cortex 17, 679-690. doi: 10.1093/cercor/ bhk016

Talsma, D., Senkowski, D., Soto-Faraco, S., and Woldorff, M. G. (2010). The multifaceted interplay between attention and multisensory integration. Trends Cogn. Sci. 14, 400-410. doi: 10.1016/j.tics.2010.06.008

Tang, X., Wu, J., and Shen, Y. (2016). The interactions of multisensory integration with endogenous and exogenous attention. Neurosci. Biobehav. Rev. 61, 208-224. doi: 10.1016/j.neubiorev.2015.11.002

Ternus, J. (1926). Experimentelle untersuchungen über phänomenale identität. Psychol. Forsch. 7, 81-136. doi: 10.1007/BF02424350

Thomas, D. R., and Jones, C. G. (1962). Stimulus generalization as a function of the frame of reference. J. Exp. Psychol. 64, 77-80. doi: 10.1037/h00 43304

Thomas, D. R., and Strub, H. (1974). Adaptation level and the central tendency effect in stimulus generalization. J. Exp. Psychol. 103, 466-474. doi: $10.1037 / \mathrm{h} 0037184$

Thomas, D. R., Svinicki, M. D., and Vogt, J. (1973). Adaptation level as a factor in human discrimination learning and stimulus generalization. J. Exp. Psychol. 97, 210-219. doi: 10.1037/h0033908

Treutwein, B., and Strasburger, H. (1999). Fitting the psychometric function. Percept. Psychophys. 61, 87-106. doi: 10.3758/BF03211951

Vatakis, A., and Spence, C. (2007). Crossmodal binding: evaluating the "unity assumption" using audiovisual speech stimuli. Percept. Psychophys. 69, 744-756. doi: 10.3758/BF03193776

Vatakis, A., and Spence, C. (2008). Evaluating the influence of the 'unity assumption' on the temporal perception of realistic audiovisual stimuli. Acta Psychol. 127, 12-23. doi: 10.1016/j.actpsy.2006.12.002

Vroomen, J., Bertelson, P., and de Gelder, B. (2001). The ventriloquist effect does not depend on the direction of automatic visual attention. Percept. Psychophys. 63, 651-659. doi: 10.3758/BF03194427

Vroomen, J., and Keetels, M. (2010). Perception of intersensory synchrony: a tutorial review. Atten. Percept. Psychophys. 72, 871-884. doi: 10.3758/APP.72.4.871

Wahn, B., and Konig, P. (2015). Audition and vision share spatial attentional resources, yet attentional load does not disrupt audiovisual integration. Front. Psychol. 6:1084. doi: 10.3389/fpsyg.2015.01084

Wallace, M. T., and Stevenson, R. A. (2014). The construct of the multisensory temporal binding window and its dysregulation in developmental disabilities. Neuropsychologia 64, 105-123. doi: 10.1016/j.neuropsychologia.2014. 08.005

Wichmann, F. A., and Hill, N. J. (2001). The psychometric function: I. fitting, sampling, and goodness of fit. Percept. Psychophys. 63, 1293-1313. doi: 10.3758/BF03194544

Conflict of Interest Statement: The authors declare that the research was conducted in the absence of any commercial or financial relationships that could be construed as a potential conflict of interest.

Copyright $(2018$ Wan and Chen. This is an open-access article distributed under the terms of the Creative Commons Attribution License (CC BY). The use, distribution or reproduction in other forums is permitted, provided the original author(s) and the copyright owner are credited and that the original publication in this journal is cited, in accordance with accepted academic practice. No use, distribution or reproduction is permitted which does not comply with these terms. 